\title{
A hazel tree relict community (Corylus avellana L., Betulaceae) from the Guadiana River Middle Basin (Ciudad Real, Spain)
}

\author{
Enrique Luengo Nicolau' ${ }^{1}$ \& Daniel Sánchez-Mata ${ }^{1}$
}

\begin{abstract}
Luengo Nicolau, E. \& Sánchez-Mata, D. A hazel tree relict community (Corylus avellana L., Betulaceae) from the Guadiana River Middle Basin (Ciudad Real, Spain). Lazaroa 36: 133-137 (2015).

A new community is proposed for the checklist of Iberian Peninsula vegetation: Chamaeirido foetidissimae-Coryletum avellanae. This forest vegetation type was discovered in the riparian landscapes along the Middle Basin of the Guadiana River (Ciudad Real, Spain), and has a true relict character. These hazel tree relict formations are seriously threatened by human activities in the study area.
\end{abstract}

Keywords: Betulaceae, Corylus avellana, Guadiana River, Iberian Peninsula vegetation.

Resumen: Luengo Nicolau, E. \& Sánchez-Mata, D. Un avellanar relíctico (Corylus avellana L., Betulaceae) en la cuenca media del Río Guadiana (Ciudad Real, España). Lazaroa 36: 133-137 (2015).

Se propone una nueva comunidad forestal para el catálogo de la vegetacíon de la Península Ibérica: Chamaeirido foetidissimae-Coryletum avellanae. Se trata de formaciones descubiertas en la cuenca media del Río Guadiana (Ciudad Real, España) que presentan carácter reliquial. Esta vegetación edafohigrófila forestal se encuentra fuertemente amenazada por la actividad humana en el área de estudio.

Palabras clave: Betulaceae, Corylus avellana, Río Guadiana, vegetación de la Península Ibérica.

Hazel tree (Corylus avellana L.) is a rare species in the landscapes on the Mediterranean Iberian Peninsula, where it always grows as infrequent specimens in hygrophilous forest communities associated with river canyons, on moist shady slopes and riverine forest fringes, among other sites (QUESADA \& al., 2009; RoDRíGUEZ GUITIÁN, 2010). There is only one reported hazel tree forest community in centralsouthern Iberian Peninsula: the supramediterranean formations of Sub-Baetic mountain territories (Albacete-Jaén provinces), corresponding to the association Geo urbani-Coryletum avellanae F. Valle, Mota \& Gómez Mercado 1986 corr. Gómez Mercado [in Rivas-Martínez \& al.] 2002 [Aceri granatensis-Quercion fagineae (Rivas Goday, Rigual \& Rivas-Martínez in Rivas Goday, Borja, Esteve, Galiano, Rigual \&
Rivas-Martínez 1960) Rivas-Martínez 1987] (cf. Rivas-MarTínez \& al., 2001, 2002). These forest formations grow on deep calcareous-dolimitic soils in canyons and narrow shady valleys.

The presence of hazel trees throughout the landscapes of Castile-La Mancha is well known. However the references to the territory of Ciudad Real are very scarce and not always sufficiently documented; we only have some unpublished technical reports (CABRERA \& FlOX, 1999/2000) and documents (GARCíA Río, 2000), in addition to other published references in collective compilations (VERDE \& al., 2000; Charco \& al., 2008; GosÁlvez \& al., 2009, among others). For some years we have been studying the area covered by hazel tree forest formations growing on steep slopes and small shady canyons in the Middle Basin of the

\footnotetext{
${ }^{1}$ Departamento de Biología Vegetal II, Facultad de Farmacia, Universidad Complutense, E-28040 Madrid, España. Email: dsmata@ucm.es
} 
Guadiana River (areas with a lower mesomediterranean thermotype, from 510 to $550 \mathrm{~m}$ asl). This forest type is certainly a relict refugee community growing on deep moist soils in the study area, an attractive natural area located between the Montes de Toledo to the north and Sierra Morena to the south, and known as Comarca de Los Montes de Ciudad Real.

Our contribution is the first documented reference to hazel tree forest formations in the territory of Ciudad Real growing in areas with a mesomediterranean thermotype; we include some herbarium vouchers conserved in the Herbarium of the Faculty of Pharmacy, Complutense University, Madrid (MAF) (see Appendix, herbarium vouchers).

Table 1 shows several relevés taken in the territories around the Guadiana River, on the El Campillo estate, in Ciudad Real, Spain, $38^{\circ} 58^{\prime} 39^{\prime}$ 'N , 4 $24^{\prime} 5^{\prime \prime} \mathrm{W}$, during the last years. This table contains eight relevés which reveal the floristic, vegetational and ecological characterisation of these peculiar formations. Some edaphohygrophilous tree species are common, such as Salix atrocinerea and Fraxinus angustifolia; along with several characteristic species such as Athyrium filix-foemina, Carex pendula, Chamaeiris foetidissima, Narcissus portensis, Osmunda regalis, and Ranunculus ficaria.

We propose to frame these hazel tree formations in a new association: Chamaeirido foetidissimae-Coryletum avellanae ass. nova hoc loco (Table 1; holotypus, relevé no. 7), within the edapho-hygrophilous forest alliance Populion albae, Fraxino angustifoliaeUlmenion minoris suballiance (see Appendix, phytosociological scheme following the proposals of Rivas-MARTíneZ \& al., 2001). These forest formations point to a dynamic community related with ash tree (Fraxinus angustifolia) and western oak (Quercus broteroi) forests developed in lowlands on deep alluvial soil; we believe they represent serial pre-forest stages or even permanent communities in highly protected and wet areas such as steep slopes, ravines and shady canyons, among others. Their biogeographical distribution covers at least the mountain territories in the Middle Guadiana River Basin (northern and eastern territories of Lusitania and Extremadura subprovince, western-Iberian Mediterranean province, westernMediterranean subregion, Mediterranean biogeographical region).

The landscape significance of this new proposed forest unit is explained by the history and dynamics of the natural vegetation in the study area. Historical references show that the potential distribution of this vegetation type was much larger than at present, when its main threats come from over-hunting and human management activities.

\section{ACKNOWLEDGEMENTS}

We would like to thank Leovigildo Flox Morales, Environmental Agent from the Autonomous Government of Castile-La Mancha for their valuable help; also Pru Brooke-Turner who revised the English language style of this contribution. 
Table 1

Chamaeirido foetidissimae-Coryletum avellanae ass. nova

(Fraxino angustifoliae-Ulmenion minoris, Populion albae, Populetalia albae, Salici purpureae-Populetea nigrae)

\begin{tabular}{|c|c|c|c|c|c|c|c|c|}
\hline Altitude (m asl) & 520 & 520 & 530 & 520 & 550 & 540 & 510 & 520 \\
\hline Area $\left(1=10 \mathrm{~m}^{2}\right)$ & 10 & 10 & 10 & 10 & 20 & 10 & 20 & 10 \\
\hline Inclination $\left(^{\circ}\right)$ & 25 & 30 & 30 & 40 & 30 & 30 & 35 & 30 \\
\hline Exposure & NW & NW & $\mathrm{NE}$ & $\mathrm{N}$ & NW & NW & $\mathrm{N}$ & $\mathrm{NE}$ \\
\hline N.N. species & 16 & 16 & 16 & 19 & 22 & 28 & 29 & 29 \\
\hline Relevé n. & 1 & 2 & 3 & 4 & 5 & 6 & 7 & 8 \\
\hline \multicolumn{9}{|l|}{ Characteristics } \\
\hline Corylus avellana & 3 & 2 & 2 & 3 & 2 & 2 & 4 & 2 \\
\hline Chamaeiris foetidissimus & 2 & 1 & 2 & 2 & 2 & 2 & 3 & 2 \\
\hline Fraxinus angustifolia & + & 1 & + & + & + & 1 & 2 & + \\
\hline Narcissus portensis & . & + & 1 & 1 & 1 & 1 & 1 & 2 \\
\hline Ranunculus ficaria & + & 1 & . & + & . & + & 1 & 1 \\
\hline Vitis sylvestis & 2 & . & . & . & 1 & 1 & 1 & 1 \\
\hline Carex pendula & 1 & 1 & . & 1 & . & . & 1 & 2 \\
\hline Salix atrocinerea & . & + & . & . & . & 1 & 2 & 1 \\
\hline \multicolumn{9}{|l|}{ Companions } \\
\hline Ruscus aculeatus & 1 & 1 & 2 & 2 & + & 3 & 2 & 1 \\
\hline Quercus broteroi & + & + & + & . & + & 1 & 1 & 1 \\
\hline Erica lusitanica & + & . & 1 & + & + & + & + & 1 \\
\hline Viburnum tinus & 1 & + & . & + & + & 1 & 1 & + \\
\hline Lonicera hispanica & 2 & . & . & 1 & 1 & 1 & 1 & 1 \\
\hline Rubus ulmifolius & . & 2 & . & + & + & + & 1 & 1 \\
\hline Osmunda regalis & 1 & . & . & + & 1 & + & + & 1 \\
\hline Smyrnium perfoliatum & . & + & . & . & + & 2 & 1 & 1 \\
\hline Phillyrea latifolia & . & . & 2 & 1 & + & 2 & 1 & . \\
\hline Crataegus monogyna & + & . & . & . & 1 & 1 & + & 1 \\
\hline Athyrium filix-foemina & . & . & . & 1 & + & + & 1 & 1 \\
\hline Rosa canina & . & + & + & . & . & 1 & 1 & + \\
\hline Carex binervis & 1 & . & . & 1 & 1 & . & 1 & + \\
\hline Lampsana communis & . & + & . & . & . & + & 1 & 1 \\
\hline Acer monspessulanum & . & . & 1 & . & + & 2 & 1 & . \\
\hline Arbutus unedo & . & + & + & . & . & 1 & 1 & . \\
\hline Paeonia broteroi & . & . & 1 & . & . & 1 & + & + \\
\hline Tamus communis & . & . & . & . & 1 & + & + & 1 \\
\hline Sibthorpia europaea & 1 & . & . & + & 1 & . & . & 2 \\
\hline Urtica dioica & + & + & + & + & . & . & . & . \\
\hline Asplenium onopteris & . & . & + & . & . & 1 & 1 & + \\
\hline Pistacia terebinthus & . & . & 1 & . & + & 1 & + & . \\
\hline Rubia peregrina & . & . & . & . & . & + & + & + \\
\hline Bryonia dioica & . & . & . & . & 1 & . & . & 1 \\
\hline Teucrium scorodonia & . & . & . & + & . & . & . & 1 \\
\hline Hypericum undulatum & . & . & . & 1 & . & . & . & 1 \\
\hline Quercus rotundifolia & . & . & + & . & . & + & . & . \\
\hline
\end{tabular}

Localities: All relevés from Ciudad Real: Luciana. Comarca de Los Montes. "El Campillo". Holotypus relevé number 7. 


\section{REFERENCES}

Cabrera, F. \& Flox, L. —1999/2000 - Inventario, catalogación y estudio florístico de los bonales de Ciudad Real. Informe técnico - Consej. Agric. Junta Com. Castilla-La Mancha, Ciudad Real. (unpubl.).

Castroviejo, S. \& al. (Eds.) - 1986- Flora iberica. Plantas vasculares de la Península Ibérica e Islas Baleares - R. Jard. Bot. CSIC, Madrid.

Charco, J., Fernández-González, F, García Río, R., Mateo, G. \& Valdés, A. - 2008- Guía de los árboles y arbustos autóctonos de Castilla-la Mancha - Cent. Inv. Amb. Mediterráneo (CIAMED), Ciudad Real.

García Río, R. — 2000 - Flora protegida y hábitats de interés de la provincia de Ciudad Real. Materiales para un curso - Consej. Agric. Junta Com. Castilla-La Mancha, Ciudad Real. (unpubl.).

Gosálvez Rey, R.U., Ayllón López, E. \& Flores Polanco, T. -2009- Fauna vertebrada terrestre en un espacio de transición biogeográfica: el borde meridional de la Meseta Sur en el entorno de Puertollano - In: Costa, M., Pieren A. \& Viejo, J.L. (Eds.). Historia Natural de Puertollano y Comarca de Calatrava. Pp. 55-93. Mem.

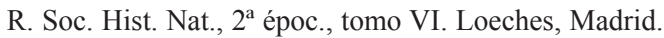

Rodríguez Guitián, M.A. — 2010 - Temperate riverside forests without alder trees in the north-west of the
Iberian Peninsula: ecology, phytosociological profile and interest for preservation policies - Lazaroa 31: 937.

Quesada, J., Valle, F. \& Salazar, C. - 2009- El paisaje vegetal ripario del río Guadalentín (Jaén-Granada, sureste de España): bases para la gestión y la conservación del medio natural — Lazaroa 30: 119-132.

Rivas-Martínez, S., Fernández-González, F., Loidi, J., Lousã, M \& Penas, A. - 2001- Syntaxonomical checklist of the vascular plant communities of Spain and Portugal to association level - Itinera Geobot. 14: 5341 .

Rivas-Martínez, S., Díaz González, T.E., FernándezGonzález, F., Izco, J., Loidi, J., Lousã, M \& Penas, A. 2002 - Vascular plant communities of Spain and Portugal (Addenda to the Syntaxonomical Checklist of 2001) - Itinera Geobot. 15(1): 5-432.

Tutin, T.G., Heywood, V.H., Burges, N.A., Moore, D.M., Valentine, D.H., Walters, S.M. \& Webb, D.A. - 19641980 - Flora Europaea. Vol. 1-5 - Cambridge Univ. Press, Cambridge.

Verde, A., Fajardo, J., Rivera, D. \& Obón, C. -2000Etnobotánica en el entorno del Parque Nacional de Cabañeros - Publ. Min. Med. Amb., Ser. Téc., Madrid. 


\section{APPENDIX}

\section{TAXONOMIC NOMENCLATURE}

The taxonomic nomenclature follows the proposal of published volumes of Flora iberica (CAstroviejo \& al., 1986), or Flora Europaea (Tutin \& al., 1964-1980). The exceptions and accepted taxonomic ranks for nomenclatural abbreviations are the following:

Lonicera hispanica: Lonicera periclymenum subsp. hispanica (Boiss. \& Reut.) Nyman Narcissus portensis: Narcissus pseudonarcissus subsp. portensis (Pugsley) A. Fern.

Quercus broteroi Rivas Mart. \& C. Sáenz

Quercus rotundifolia Lam.

Vitis sylvestris: Vitis vinifera subsp. sylvestris (C.C. Gmel.) Hegi

HERBARIUM RECORDS

Chamaeiris foetidissima (L.) Medik.

Ciudad Real: Luciana. Comarca de Los Montes. Relic hazel forest close to the Guadiana river in “El Campillo", 3858’39”N, 04²4’05”W, 535 m asl., 10.06.2012, E. LuENGO (MAF 175041).

\section{Corylus avellana $\mathrm{L}$.}

Ciudad Real: Luciana. Comarca de Los Montes. Relic hazel forest close to the Guadiana river in “El Campillo", 3858’39”'N, 04²4'05”W, 535 m asl., 10.10.2012, E. LuENGO (MAF 175042).

\section{PhytosociologicAl SCHEME}

Salici purpureae-Populetea nigrae (Rivas-Martínez \& Cantó ex Rivas-Martínez \& al. 1991) Rivas-Martínez \& al. 2001

Populetalia albae Br.-Bl. ex Tchou 1948

Populion albae br.-Bl. ex Tchou 1948

Fraxino angustifoliae-Ulmenion minoris Rivas-Martínez 1975

1. Chamaeirido foetidissimae-Coryletum avellanae ass. nova 